\section{Schedule-induced alcohol ingestion}

PETER B. EVERETT and RICHARD A. KING, University of North Carolina, Chapel Hill, N.C. 27514

Rats were run on a behavioral schedule that produced excessive collateral Kool-Aid ingestion. Ethyl alcohol was then mixed with the Kool-Aid in varying concentrations. The rats continued to drink excessive amounts of fluid, and the resultant quantity of alcohol ingestion was high.

An experimental animal that voluntarily ingests alcohol is most desired for studies simulating human alcoholism, but such a preparation is difficult to obtain. Most researchers resort to force feeding or intraperitoneal injections of alcohol. Senter, Smith, \& Lewin (1967) have made rats ingest alcohol by defining the drinking as an operant that either avoids shock or delivers food. Although a step closer to voluntary ingestion, their study still maintains strong contingencies over the drinking behavior (i.e., to get food or avoid shock the Ss must drink).

Animals working on food-pellet reinforced interval schedules in which the reinforcement availability is over $30 \mathrm{sec}$ often develop schedule-induced polydipsia (Falk, 1961) or excessive collateral water drinking. It is not uncommon to have a $250-\mathrm{g}$ rat ingest $100 \mathrm{ml}$ of water during a 3h test session. Falk (1967) interprets this behavior as displacement activity, whereas others call it a superstitious operant (Clark, 1962). Regardless of how this collateral water drinking is classified, it is a method for obtaining an experimental animal that ingests large volumes of water.

In the present study, we introduced alcohol into a Kool-Aid solution for animals on a behavioral schedule that produces polydipsia. Under such a procedure, it is possible to present low concentrations of alcohol and induce a high level of alcohol ingestion because of the large fluid intake. A feature of the polydipsic paradigm of alcohol ingestion is that the drinking behavior is "voluntary"-there are no arbitrary E-controlled contingencies. This is possibly a situation more analogous to human alcohol consumption.

\section{SUBJECTS}

Two male Sprague-Dawley rats, approximately 200 days old, were used as

Fig. 1. Median total fluid intake per session under various experimental conditions.
Ss. They were reduced from their free-feeding weights of $330 \mathrm{~g}$ and $340 \mathrm{~g}$ to the experimental weight of $280 \mathrm{~g}$.

$$
\text { APPARATUS }
$$

A standard Scientific Prototype rat environmental test chamber was used. A drinking spout, a pellet dispenser cup, and a white "session on" light were mounted on the front panel. The chamber's grid floor was a common to a Grason-Stadler drinkometer circuit. The other pole was the metal drinking spout. Noyes $45-\mathrm{mg}$ rat pellets were delivered automatically.

The behavioral programming was accomplished by a series of relays and timers. All events, including licks from the drinkometer circuit, were recorded on counters and a cumulative recorder. PROCEDURE

During the diet rationing, the Ss had free access to water in their home cages. When the experimental weight was reached, the water was replaced by grape Kool-Aid. Ad lib Kool-Aid was available for 5 more days in the home cage as the Ss' body weights were maintained constant at $280 \mathrm{~g}$. At the end of the 5th day, the Kool-Aid was taken away.

The next day, and throughout the rest of the experiment, the Ss were run daily in the test chamber. During the first test session, only ad lib Kool-Aid was available. All test sessions lasted $100 \mathrm{~min}$. Each S's absolute fluid intake as well as total "licks" were recorded daily.

The next test sessions included the free presentation of the Noyes rat pellet at a
VI 1-min. No operants were required for the delivery of the pellets.

As collateral Kool-Aid drinking developed and then stabilized, the next manipulation was made. This was the addition of $100 \%$ ethyl alcohol (ETOH) to make a $1 \%$ solution with the Kool-Aid. When the drinking stabilized at each ETOH percentage, the amount of alcohol was increased to a higher dosage. The solutions tested were $1 \%, 2 \%, 3 \%, 4 \%$, and $5 \%$. During the entire study, the test feeding of pellets was supplemented in the home cage with sufficient food to maintain a $280-\mathrm{g}$ body weight.

\section{RESULTS AND DISCUSSION}

Figure 1 shows the median total fluid intake for the last 3 days in each experimental condition. The "no pell" condition is the test day before the free pellets were dropped into the experimental chamber. The Ss in this situation drank from $10 \mathrm{ml}$ to $14 \mathrm{ml}$ of unadulterated Kool-Aid. The second condition in Fig. 1 , labeled "0\% ETOH," illustrates drinking at polydipsic levels as the pellets were dropped into the experimental chamber. S1 drank a median of $42 \mathrm{ml}$ of Kool-Aid in this condition, and $\mathbf{S} 2$ had a median intake of $50 \mathrm{ml}$. The rest of the conditions in Fig. 1 are the same as the "O\% ETOH" condition except for the addition of a given per cent ETOH into the Kool-Aid. Polydipsic levels of fluid intake were maintained throughout all of the conditions that included alcohol in the fluid.

Figure 2 depicts the median quantity of $100 \%$ ETOH that was ingested during a session under each of the experimental conditions (i.e., $1 \%, 2 \%, 3 \%, 4 \%$, or $5 \%$

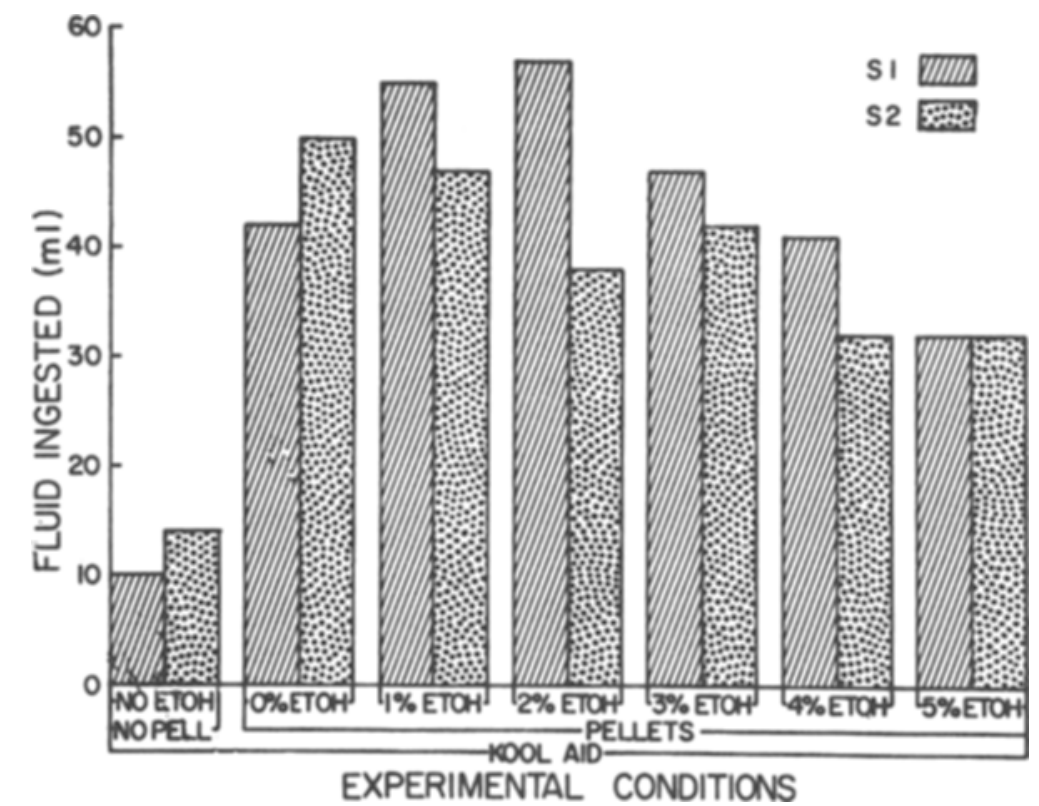




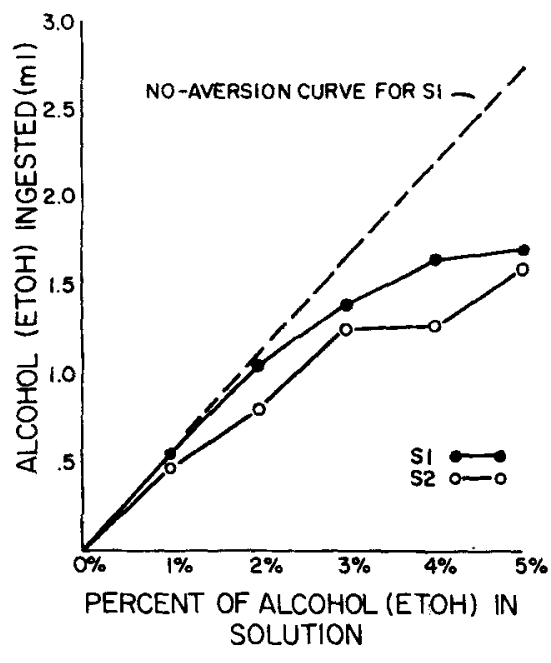

Fig. 2. Median amount of absolute alcohol ingested in a $100-\mathrm{min}$ session with a given alcohol concentration.

ETOH in solution). The greatest amount of alcohol was ingested at the $5 \%$ solution. At this concentration, S1 drank $1.7 \mathrm{ml}$ of ETOH, and $\mathrm{S} 2$ drank $1.6 \mathrm{ml}$. This increasing amount of absolute alcohol ingestion was a factor of an increasing per cent of alcohol in solution and not a greater polydipsic level of drinking as Fig. 1 demonstrates. A no-aversion plot has been made on Fig. 2. This is a representation of what the absolute ETOH intake would be if $S 1$ had maintained a constant level of polydipsic drinking throughout the entire experiment.

Subjectively, the $S$ s appeared intoxicated at all solutions equal to or greater than $2 \%$ ETOH. They would often be asleep on the floor of the experimental chamber in a nontypical "sprawled" fashion after the session was over. Their breath smelled of alcohol, and they lacked a placing response.
Lester (1961) reported a similar study. Rats drank $9.9 \mathrm{ml}$ of a $5.6 \%$ alcohol solution in $1 \mathrm{~h}$ on a VI $55-\mathrm{sec}$ food schedule. Lester maintained that the bar press for the food pellet was a necessary requirement if measurable levels of alcohol ingestion were to be observed. The present study is in direct contradiction to this. Food pellets were dropped into the experimental chamber at variable intervals without operant contingencies, and alcohol ingestion levels nearly twice those reported by Lester were found.

This experiment is presented as a different method of experimentally obtaining high alcohol intake in subhuman Ss. The results are encouraging. This study is by no means meant to be an exhaustive or extremely exacting quantification of the parameters involved in "schedule-induced alcohol ingestion." Percentages of alcohol greater than $5 \%$ certainly warrant investigation as do observations of amount of addiction or dependence on alcohol. This is primarily a "pilot" study in which it has been shown that rats will ingest large amounts of alcohol in situations in which they are not coerced to do so.

\section{REFERENCES}

CLARK, F, C. Some observations on the adventitious reinforcement of drinking under food reinforcement. Journal of the Experimental Analysis of Behavior, 1962, 5, 61-63.

FALK, J. L. Production of polydipsia in normal rats by a intermittent food schedule. Science, 1961, 133, 195-196.

FALK, J. L. Conditions producing psychogenic polydipsia in animals. Paper presented at the New York Academy of Sciences Conference on Neural Regulation of Food and Water Intake, New York, February 1967.

LESTER, D. Self-maintenance of intoxication in the rat. Quarterly Journal of Studies on Alcohol, 1961, 22, 223-231.

SENTER, R. J., SMITH, F. W., \& LEWIN, S. Ethanol ingestion as an operant response. Psychonomic Science, 1967, 8, 291-292. retention deficit compared to the controls. In another experiment, two groups of 16 fish were given 4 days of training and were then injected immediately with puromycin or saline. There were indications of interference in this experiment also. These experiments are at variance with recent hypotheses that puromycin interferes with the consolidation stage of memory.

The inhibitory effects of puromycin on memory have been studied in a long series of experiments starting in 1963 (Flexner, Flexner, \& Stellar, 1963; Agranoff, Davis, \& Brink, 1965). In a series of experiments with goldfish, Agranoff and his colleagues have shown that puromycin interferes with the retention of a learned shock-avoidance response when injected intracranially over the brain. This effect is obtained only when puromycin is injected immediately after the learning phase, presumably before the consolidation process has taken place. Puromycin injected $1 \mathrm{~h}$ or more after learning has little effect on retention. When puromycin is injected before the learning period, no effect is seen in learning the avoidance response, but a deficit in retention appears, however, when these animals are tested 3 days later.

The results of recent experiments by Potts \& Bitterman (1967) suggested that the locus of this interference is the consolidation of conditioned fear. The purpose of the experiments described below was to explore further the nature of this interference by using a classical-conditioning procedure instead of the shuttlebox avoidance.

\section{METHOD}

Four experiments were conducted with the fish classical-conditioning apparatus described by Longo \& Bitterman (1960). Briefly, this apparatus consists of fully automated chambers where light and shock are paired, and the response (activity) is monitored by a paddle connected to a phonograph cartridge. The output of the cartridge is amplified and converted to relay pulses.

In each of the first three experiments, two groups of goldfish (16 in each group), measuring 8 to $10 \mathrm{~cm}$ (from snout to base of caudal peduncle), were given 60 lightshock pairings in a single 1 -h session. In the first 50 trials, a 10-sec light was followed by a brief .1-sec mild ac electric shock. In the last 10 trials of each session, the CS-US interval was increased to $20 \mathrm{sec}$. One group in each of the experiments was injected intracranially by Agranoff's technique with 200 micrograms of puromycin hydrochloride in 10 microliters of saline. The control groups of each experiment were injected with 10 microliters of saline alone.
Düsseldorf, Germany, and NICHOLAS LONGO, Colgate University, ${ }^{2}$ Hamilton, N.Y. 13346

Ninety-six goldfish, divided into six groups, were trained in a classical conditioning experiment in a single hour-long session. Three of the groups were injected intracranially over the brain with puromycin 0,24 , and $48 \mathrm{~h}$ after training, the three control groups were injected with saline. Tested 3 days after training, the puromycin groups all showed a marked 